\title{
A Retrospective Analysis of Outcome of Vitrified vs Fresh Oocytes among Donor and Patient Cohorts
}

\author{
Rutvij J Dalal ${ }^{1}$, Akanksha P Mishra² $^{2}$ Sahil Gupta ${ }^{3}$, Divya Rani ${ }^{4}$
}

\begin{abstract}
Background: Oocyte cryopreservation has been an enigma for many years. It has not been easy to freeze the largest cell of the body owing to its large water content and low surface-to-volume ratio. The recovery of cryopreserved oocytes, for many years, remained dismal at best due to ice crystal formation and insufficient cryoprotectant permeation. In recent years, particularly after the advent of vitrification, a much more successful cryopreservation recovery has been achieved, and oocyte cryopreservation has now become a mainstream treatment procedure in artificial reproduction technology (ART).

Aim and objective: Our study aims to retrospectively analyze oocyte cryopreservation data collected over a period of 5 years, between two centers, with a standardized vitrification-warming technique. The parameters taken into consideration were oocyte survival, fertilization, blastocysts formation, implantation rate, and live birth rate.

Result: We have observed no significant difference in fresh vs frozen oocytes in the donor as well as patient cohorts in terms of implantation rates and live birth outcomes. Our data have been very encouraging, so that we can offer oocyte cryopreservation to the desiring women seeking this technology, and have opened up a plethora of opportunities with the successful application of oocyte vitrification.

Conclusion: We are attempting a further, more elaborate study with much bigger sample size and many more individual criteria to follow-up the success of this technique more closely.

Keywords: Fertility, Fertility preservation, Intracytoplasmic sperm injection, IVF ET, Oocyte cryopreservation, Reproductive health, Retrospective study, Vitrification, Vitrified oocyte.

International Journal of Infertility and Fetal Medicine (2021): 10.5005/jp-journals-10016-1210
\end{abstract}

\section{INTRODUCTION}

With recent advances in science all over the world, artificial reproduction technology (ART) too underwent fairly quick upgradation from simple IVF for fallopian tube disorders to a range of treatments addressing the varied causes of infertility in both females and males. One of such advancements is vitrification, which revolutionized cryopreservation outcomes, thereby allowing for a lot of flexibility and improved results. After somewhat dismal survival rates achieved by the slow freezing method, cryopreservation did not become mainstream for a good many years. Vitrification surpassed the drawbacks of slow freezing and proved to be a very reliable component of ART. ${ }^{1}$ Vitrification was first tried for embryos and sperms but it was neglected for oocytes for several years because of the challenges faced. Significant advancements in the vitrification procedure made it more suitable for oocyte cryopreservation. Since then, success rates of oocyte freezing have risen making it widely useful. ${ }^{2}$ Oocyte freezing helps in avoiding legal and ethical restrictions associated with excess embryo freezing. It saves the oocytes in case of unavailability of sperm samples on the day of oocyte retrieval. However, oocyte freezing held many further purposes to be fulfilled, it was emphasized to bring vitrification to a level where it can be applied to oocyte cryopreservation too. Currently, oocyte freezing is utilized for fertility preservation. Fertility preservation for medical reasons: women undergoing gonadotoxic treatment, and social reasons, i.e., women opting for a delay in motherhood and who do not want to experience age-related fertility decline. Oocyte freezing also helps in establishing egg banks so that patients with oocyte donation programs can be benefitted better.

\author{
${ }^{1}$ Department of IVF, Janini IVF, New Delhi, India \\ ${ }^{2}$ Department of IVF, Lilavati Hospital and Research Center, Mumbai, \\ Maharashtra, India \\ ${ }^{3}$ Department of IVF, Aveya Fertility, New Delhi, India \\ ${ }^{4}$ Department of Clinical Embryology, Janini IVF, New Delhi, India \\ Corresponding Author: Rutvij Jay Dalal, Department of IVF, Janini IVF, \\ New Delhi, India, Phone: +91 9930273377, e-mail: rutvij.dalal@gmail. \\ com \\ How to cite this article: Dalal RJ, Mishra AP, Gupta S, et al. A \\ Retrospective Analysis of Outcome of Vitrified vs Fresh Oocytes among \\ Donor and Patient Cohorts. Int J Infertil Fetal Med 2021;12(1):1-6. \\ Source of support: Nil \\ Conflict of interest: None
}

Success rates in oocyte freezing might be subjective. Due to the big size and vulnerability to both physical and chemical stress, oocyte freezing requires a scientifically sound and well-experienced embryologist. However, for the past few years, oocyte vitrification has also been accepted widely and used for both infertile and fertile women.

This study is a retrospective analysis of outcomes of cryopreserved oocytes in terms of survival, fertilization, blastocyst development, implantation rate, and live births in comparison with fresh oocytes. The results are encouraging and allow us to broaden the application of this exciting technique. However, it will be prudent to conduct larger studies and follow-up on long-term developmental consequences of children conceived via oocyte cryopreservation. 


\section{History of Oocyte Cryopreservation}

The first successful pregnancy from cryopreserved oocytes was reported to be Chen in $1986 .{ }^{3}$ The initial method of cryopreservation was a slow one with the addition of dimethyl sulfoxide as the cryoprotectant in a single step. The temperature was slowly reduced till $-7^{\circ} \mathrm{C}$, the ice nucleation point, and further till $-36^{\circ} \mathrm{C}$. Following that the temperature was rapidly decreased to $-196^{\circ} \mathrm{C}$ and the cryodevice was then plunged into liquid nitrogen. To achieve thawing before fertilization, the oocytes were rapidly warmed to $37^{\circ} \mathrm{C}$ in a water bath and exposed to reducing concentrations of the cryoprotectant. ${ }^{4}$

However, the initial attempts to cryopreserve oocytes were wrought with difficulties owing to the low surface area to volume ratio as well as the tendency of intracellular ice crystal formation leading to cytoplasmic organelle damage. The success of earlier oocyte cryopreservation techniques could not be judged by no visible cryodamage alone, as it was reported that the cryopreservation technique itself might be responsible for destabilizing microfilaments and microtubules in mammalian oocytes. Furthermore, the issues of zona hardening and low fertilization were observed postoocyte cryopreservation. ${ }^{5}$

A more reliable method of oocyte cryopreservation eluded scientists across the world till 1999 when the first live birth from vitrified-thawed oocytes was reported. Vitrification involves a much higher concentration of permeable and nonpermeable cryoprotectants that prevent ice crystal formation, followed by ultra-rapid cooling achieved by direct plunging into liquid nitrogen. The warming is achieved equally rapidly by directly plunging the cryopreserved oocytes from $-196^{\circ} \mathrm{C}$ into warming media at $37^{\circ} \mathrm{C}$ followed by step-wise exposure to reducing the concentration of cryoprotectants. $^{6}$

By 2013, enough data were generated on oocyte cryopreservation outcomes that ASRM moved to provide the status of mainstream treatment to oocyte cryopreservation as opposed to the "experimental" it was earlier. In 1999, approximately 100 cryopreserved oocytes were needed to achieve one pregnancy, ${ }^{7}$ by 2013 , only 20 vitrified oocytes were required. ${ }^{8}$

Oocyte cryopreservation still remains an elusive skill, where nuances make all the difference in the outcome. A good cryopreservation medium, skilled embryologist, and strict adherence to the protocol are vital to successful oocyte cryopreservation.

\section{Challenges Faced during Oocyte Cryopreservation}

As previously stated, oocyte cryopreservation is quite a challenge owing to the high volume of the oocyte and its susceptibility to form intracellular ice crystals due to high water content. It is the largest cell of the body and the largest cell to be successfully cryopreserved.

Oocytes have a surface area to volume ratio of 0.05 , and for this reason, they take much longer to reach osmotic equilibrium as compared to sperm whose ratio is $4.3 .^{9}$ The nonpermeable cryoprotectants take longer to dehydrate the cell and the permeable cryoprotectants take longer to permeate the oocyte. During the osmotic events, the oocyte experiences extreme fluctuations in cell volume, which may lead to membrane or organelle damage, thus adversely affecting the clinical outcome. ${ }^{10}$ The cryoprotective agents (CPAs) have to be added in a gradual and precise manner to minimize cell damage. The CPAs create an osmotic gradient assisting the dehydration of the cell, they permeate the cell to convert the cytoplasm into an amorphous state and reduce the temperature of crystallization, they also stabilize the intracellular membranes and structures to prevent them from cryoinjury. ${ }^{11}$

In this study, all the oocytes have been vitrified at the MII stage where oocyte maturation is complete. The more obvious damage to the oocyte due to cryopreservation may be membrane damage, intracellular crystal formation, or osmotic shock. There may be other sublethal damages that may not be apparent but may lead to a subclinical outcome, like zona hardening, damage to the meiotic spindle or other intracellular organelles, ${ }^{12}$ in vitro aging of the oocyte, or other epigenetic changes.

\section{Materials and Methods}

The retrospective study conducted at two IVF centers (Aveya IVF and Fertility Centre and Janini IVF) includes all oocyte cryopreservation done over a period of 5 years (2015-2019). To circumvent any variation of technique resulting in differences in outcome, all the freeze-thaw and intracytoplasmic sperm injection (ICSI) cycles accounted for in this study, were performed by a single embryologist. The cohorts have been divided into autologous and donor.

Women in the autologous cohort were the ones undergoing the IVF cycle, but their oocytes could not be fertilized on the day of oocyte collection due to the following reasons:

- Unavailability of sperm.

- Oocyte pooling program.

- Poor sperm parameters, wherein the patient wanted to inseminate few oocytes to first assess the outcome with partner's sperm and freeze the rest to eventually have the option to use biopsy/donor sperm without having to undergo another ovarian stimulation.

- Social oocyte freezing.

The second cohort was donor oocytes which were frozen for the sake of quarantine and proper recipient matching.

\section{Ovarian Stimulation}

The women underwent ovarian hyperstimulation by using either an antagonist protocol involving $\mathrm{GnRH}$ antagonist or $\mathrm{GnRH}$ agonist for pituitary suppression. The ovaries were stimulated by recombinant FSH and purified hMG mixed protocol. The dose of gonadotropins employed varied from 150 to $225 \mathrm{IU}$ individualized based on patient parameters like antral follicle count, BMI, and serum AMH levels. The final maturation trigger was $4 \mathrm{mg} \mathrm{GnRH}$ agonist (antagonist stimulation protocol) or 10,000 IU hCG. Post 36 hours of the trigger, oocyte collection under anesthesia was performed.

\section{Oocyte Freezing}

The oocytes were incubated for an hour post-collection and before vitrification. They were then denuded using $80 \mathrm{IU} / \mathrm{mL}$ hyaluronidase and further in gamete buffer. Vitrification was performed no more than 2 hours post-egg collection.

The protocol used was as suggested by Kuwayama using Kitazato medium. Oocytes were put into base vitrification solution and then over a period of 9 minutes, gradually exposed to increasing concentration of cryoprotectants in the equilibration solution. The oocytes were then exposed to vitrification solution and within 60 seconds, loaded on the Cryotop device, and dipped in liquid nitrogen, thus achieving a cooling rate of $>20,000^{\circ} \mathrm{C} /$ minute. Two to three oocytes were vitrified per device. The devices were 
stored at $-196^{\circ} \mathrm{C}$ in liquid nitrogen. Although few concerns have been raised regarding the possibility of cross-infection in an open vitrification system, we have never come across such an incident in our setup. The open vitrification method was applied in all oocyte cryopreservation.

Thawing or warming of oocytes was performed by directly plunging the oocytes from the liquid nitrogen to the thawing solution ( $1 \mathrm{M}$ sucrose) at $37^{\circ} \mathrm{C}$. After 1 minute, the oocytes were transferred to dilution solution ( $0.5 \mathrm{M}$ sucrose) for 3 minutes, followed by washing solution (no cryoprotectant) for 7-8 minutes, thus exposing the oocytes to a series of reducing cryoprotectant concentration.

The oocytes were incubated for 2 hours before performing ICSI.

\section{Embryo Culture}

The oocytes were cultured in a trigas benchtop incubator $\left(5 \% \mathrm{O}_{2}\right.$, $\left.6 \% \mathrm{CO}_{2}, 89 \% \mathrm{~N}_{2}\right)$. The fertilization was noted the next day (16-18 hours post-ICSI). The embryos were assessed on day 2, 3, and 5 and sometimes till day 6 . Strictly two blastocysts were transferred per patient per cycle. Any extra embryos were vitrified and stored. Three or more blastocysts were never transferred, and the patients requesting single embryo transfer have been excluded from the data for sake of statistical simplicity.

The patients were followed up for implantation results and live birth outcomes.

\section{Statistical Analysis}

The data were entered using MS-Excel-2007 and analyzed using SPSS-16 software. Descriptive analysis for categorical data was expressed as frequency and percentage (Figs 1 to 4).

Chi-square test is used to compare proportions between two groups to find out the significance and $p$ value $<0.05$ was taken as statistically significant (Tables 1 and 2).

\begin{tabular}{lll}
\hline & Frozen oocytes & Fresh oocytes \\
\hline Donors & 1,089 & 2,552 \\
Patients & 437 & 3,107 \\
\hline
\end{tabular}

\section{Result and Discussion}

This study took into account the very basic criteria of oocyte survival post-thaw followed by a comparison between fresh

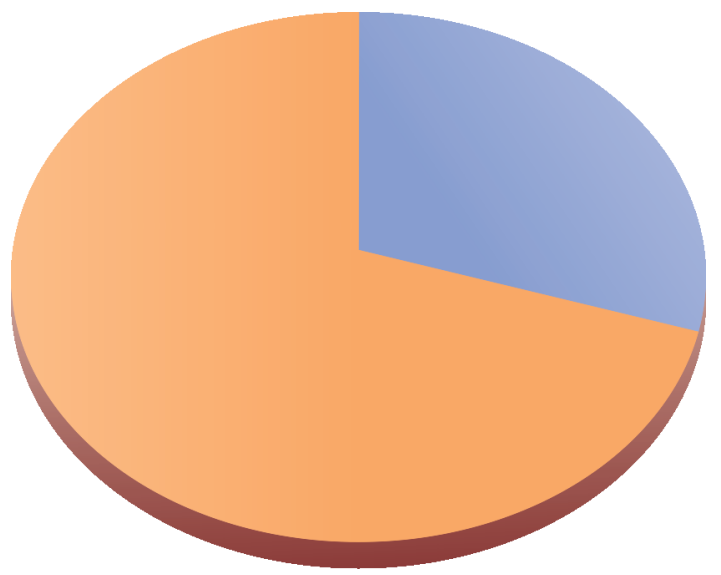

Frozen oocytes

Fresh oocytes

Fig. 1: Number of fresh vs frozen oocytes in the donor group and vitrified oocytes in terms of key outcome measures such as fertilization rate, number of blastocysts generated, overall implantation rate, and live births. The aim was to determine if oocyte cryopreservation may be considered an effective mainstream treatment in ART. The donor category was analyzed separately to especially rule out any bias arising from patient age and inherent subfertility issues like oocyte quality, which are expected to be present in the patient group.

A total of 1,404 oocytes were vitrified in the donor group, out of which 1,112 were thawed. One thousand and eighty-nine oocytes survived the cryopreservation-warming procedure which forms an encouraging $97.9 \%$ survival rate. These oocytes were then subjected to ICSI. The total number of fresh mature oocytes subjected to ICSI was 2,552 . No significant difference was seen in the fertilization rate of vitrified $v$ s fresh oocytes. A statistically significant difference was, however, observed in the total number of blastocysts generated, with the number being lower in the frozen oocytes category. Not all the blastocysts were transferred, as the total number of blastocysts transferred per patient was a constant two with no exceptions. There was no significant difference observed between implantation rate and live birth rate between the fresh and frozen cohorts in the donor group.

In the patient category, 538 oocytes were vitrified, of which 462 were warmed as per requirement. The oocyte survival rate was $94.5 \%$ with 437 oocytes surviving the cryopreservation. A statistically significant difference was seen in the fertilization rate when compared with 3,107 fresh oocytes subjected to ICSI in the patient group. However, blastocyst formation rate, implantation rate, or live birth rate were comparable in the fresh $v$ s frozen cohorts in the patient group.

The result of vitrification of oocytes in this retrospective analysis looks promising with the number of oocytes required for live birth in cryopreserved oocytes approaching that of fresh.

A successful oocyte cryopreservation program opens up a new range of possibilities in the realm of fertility treatments. Effective egg banking programs may be established for female fertility preservation not catering solely to women planning to get pregnant in the near future. It may greatly benefit the following nonconventional groups.

- Those with legal or religious obligation against embryo freezing: Certain countries prohibit freezing of embryos citing moral

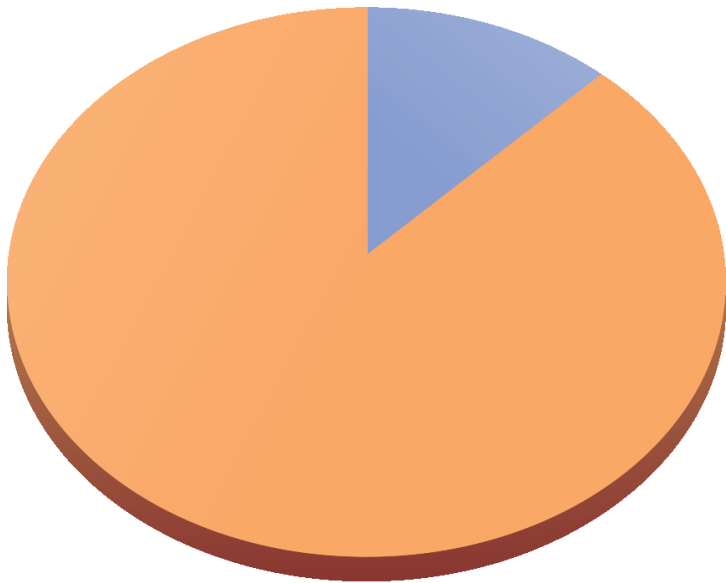

Frozen oocytes

Fresh oocytes

Fig. 2: Number of fresh vs frozen oocytes in the patient group 


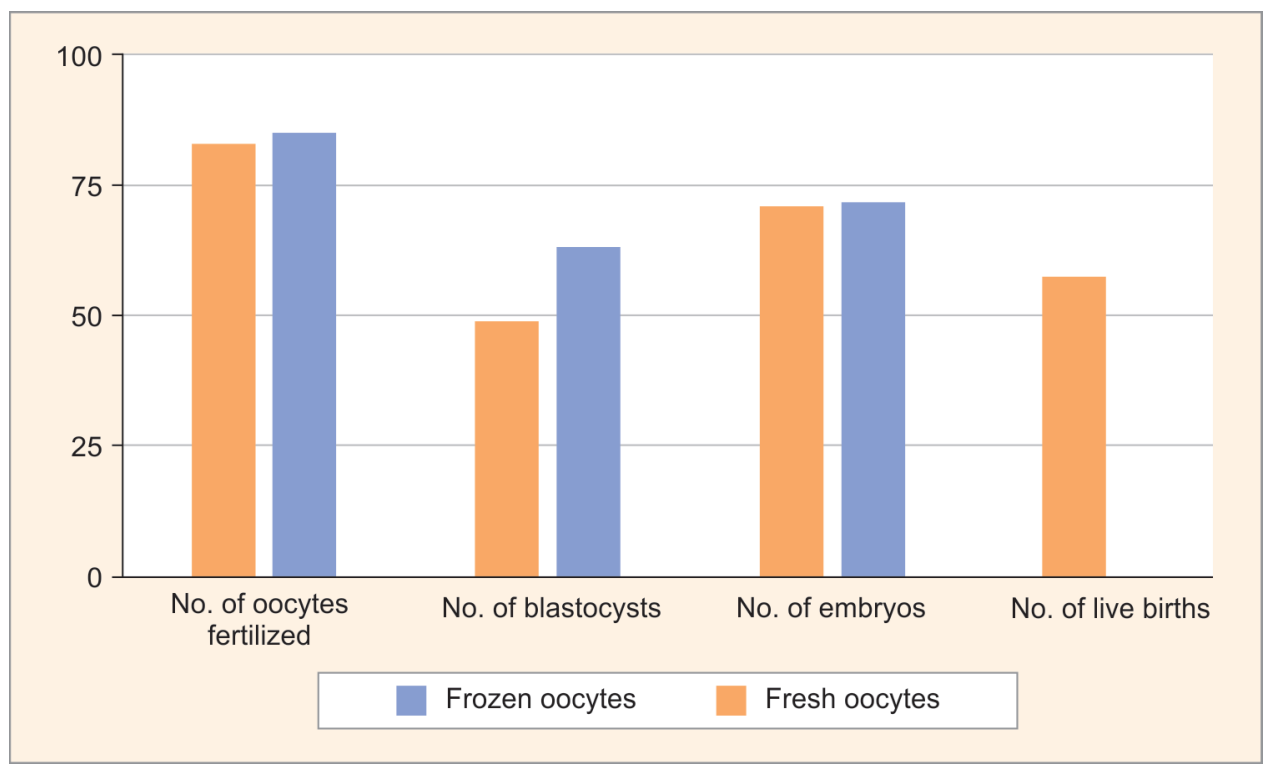

Fig. 3: Comparison of outcome between fresh vs frozen oocytes in the donor group

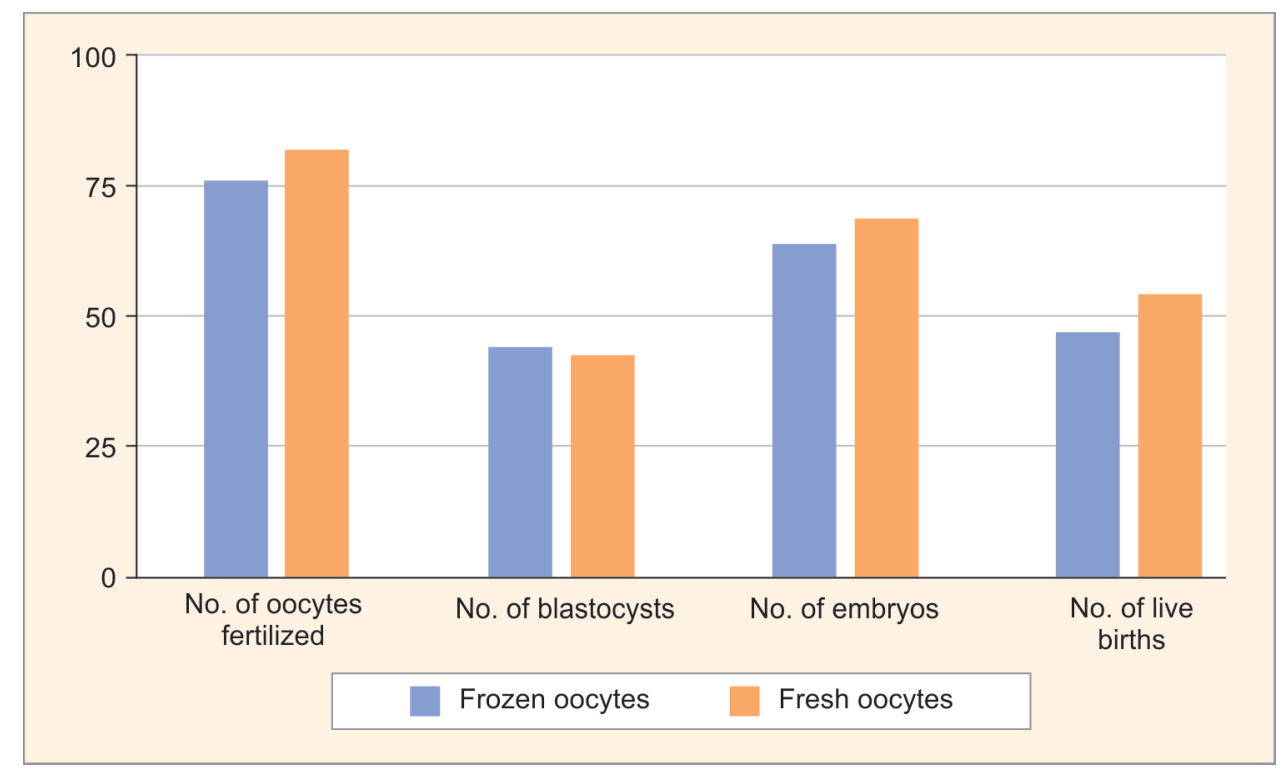

Fig. 4: Comparison of outcome between fresh vs frozen oocytes in the patient group

Table 1: Outcome of fresh vs vitrified oocytes in the donor group

\begin{tabular}{lllll}
\hline For donors & Frozen oocytes & Fresh oocytes & $p$ value & Significance \\
\hline Total no. of mature oocytes & 1,404 & 2,552 & - & - \\
No. of oocytes thawed & 1,112 & - & - & - \\
No. of oocytes survived & $1,089(97.9 \%)$ & - & - & - \\
No. of oocytes fertilized & $904(83.0 \%)$ & $2,170(85.0 \%)$ & 0.123 & Not significant \\
No. of blastocysts formed & $532(48.9 \%)$ & $1,619(63.4 \%)$ & $<0.001$ & Significant \\
No. of blastocysts frozen & 44 & 641 & - & - \\
Total no. of blastocyst transferred & 488 & 978 & - & - \\
Blastocysts transferred/patient & 2 & 2 & - & - \\
Total no. of embryos implanted & $346(70.9 \%)$ & $704(71.9 \%)$ & 0.665 & Not significant \\
Total no. of live births & $280(57.4 \%)$ & $577(58.9 \%)$ & 0.553 & Not significant \\
\hline
\end{tabular}

(Chi-square test) $(p<0.05-$ Significant) 
Table 2: Outcome of fresh vs vitrified oocytes in the patient group

\begin{tabular}{lllll}
\hline For patients & Frozen oocytes & Fresh oocytes & p value & Significance \\
\hline Total number & 538 & 3,107 & - & - \\
No. of oocytes thawed & 462 & - & - & - \\
No. of oocytes survived & $437(94.5 \%)$ & - & - & - \\
No. of oocytes fertilized & $333(76.2 \%)$ & $2,542(81.8 \%)$ & 0.004 & Significant \\
No. of blastocysts formed & $193(44.2 \%)$ & $1,324(42.6 \%)$ & 0.539 & Not significant \\
No of blastocysts frozen & 13 & 222 & & \\
Total no. of blastocyst transferred & 180 & 1,102 & - & - \\
Blastocysts transferred/patient & 2 & 2 & & Not significant \\
Total no. of embryos implanted & $115(63.9 \%)$ & $760(68.9 \%)$ & 0.175 & Not significant \\
Total no. of live births & $84(46.7 \%)$ & $598(54.3 \%)$ & 0.058 &
\end{tabular}

(Chi-square test) $(p<0.05-$ Significant)

dilemmas regarding the status of the embryo. ${ }^{13}$ Similarly, some individual beliefs also at times do not allow embryo freezing. Oocyte vitrification might eliminate the ambiguity by allowing the cryopreservation of gametes instead of an embryo.

- Oncologic patients: Patients undergoing gonadotoxic oncotherapy will be able to preserve their fertility with the help of oocyte cryopreservation. ${ }^{14}$

- Other medical conditions: Women at risk of premature ovarian failure or aggressive endometriosis may look at oocyte cryopreservation as a viable fertility preservation option till they find a suitable partner. ${ }^{15}$

- Social cryopreservation: Women who wish to delay childbearing for personal reasons may electively cryopreserve their oocytes. ${ }^{16}$

- Donor oocyte banking: Donor oocyte cryopreservation will give a lot of flexibility to the treating doctor in assigning the closest matched oocytes to the recipient. A database may be created with the characteristics of the donor and matched with the recipient overcoming the challenge of availability and synchronization of the most suitable donor. An efficient allocation of oocytes to a recipient from a pool of oocytes reduces the cost incurred as well as gives the added advantage of re-testing donors after a quarantine period thus elimination any inadvertent infection transmission. ${ }^{17}$

- Gender reassignment surgery: A section of individuals undergoing gender reallocation surgery might wish to preserve their fertility by cryopreserving their oocytes. ${ }^{18}$

\section{Conclusion}

This study is among the largest in terms of data since it spans a period of almost 5 years. We have had very encouraging results in terms of fertilization and implantation percentage as well as live births. This study bolsters the technique of oocyte vitrification as the mainstream option for social oocyte cryopreservation or donor oocyte quarantine. It may also serve to save an entire ovarian stimulation cycle in the absence of sperm on the day of egg collection. ${ }^{19}$

Additionally, we have also cryopreserved oocytes of oncologic patients who have not yet availed the fertilization of their eggs.

As with any treatment modality, it is wise to collect more and more data to acquire additional knowledge of its outcome. It is important to note that the data collected in this study is limited to a particular team. More multicentric trials may be necessary to compare outcomes leading to process optimization and limited variability. Standardization of protocol and universalizing of oocyte vitrification skills might lead to the requirement of less number of oocytes needed to achieve a live birth.

What is beyond the scope of this study though, is the possibility of any epigenetic modifications induced by the process of oocyte vitrification. The limited follow-up of babies born through vitrified oocytes suggests no difference in parameters as compared to babies born through normal conception. However, it will be interesting to follow the progress of these children through adulthood to ensure the complete safety of this procedure.

\section{References}

1. Edgar DH, Gook DA. A critical appraisal of cryopreservation (slow cooling versus vitrification) of human oocytes and embryos. Hum Reprod Update 2012;18(5):536-554. DOI: 10.1093/humupd/dms016.

2. Kuwayama M, Vajta G, Kato O, et al. Highly efficient vitrification method for cryopreservation of human oocytes. Reprod Biomed Online 2005;11(3):300-308. DOI: 10.1016/S1472-6483(10)60837-1.

3. Chen C. Pregnancy after human oocyte cryopreservation. Lancet 1986;1(8486):884-886. DOI: 10.1016/S0140-6736(86)90989-X.

4. Leibo SP, Songsasen N. Cryopreservation of gametes and embryos of non-domestic species. Theriogenology 2002;57(1):303-326. DOI: 10.1016/S0093-691X(01)00673-2.

5. Kopeika J, Thornhill A, Khalaf Y. The effect of cryopreservation on the genome of gametes and embryos: principles of cryobiology and critical appraisal of the evidence. Hum Reprod Update 2015;21(2):209227. DOI: $10.1093 /$ humupd/dmu063.

6. Simopoulou M, Asimakopoulos B, Bakas P, et al. Oocyte and embryo vitrification in the IVF laboratory: a comprehensive review. Folia Med (Plovdiv) 2014;56(3):161-169. DOI: 10.2478/folmed-20140023.

7. Porcu E. Freezing of oocytes. Curr Opin Obstet Gynecol 1999;11(3):297300. DOI: 10.1097/00001703-199906000-00011.

8. Donnez J, Dolmans MM. Fertility preservation in women. Nat Rev Endocrinol 2013;9(12):735-749. DOI: 10.1038/nrendo.2013. 205.

9. Gilmore JA, Liu J, Gao DY, et al. Determination of optimal cryoprotectants and procedures for their addition and removal from human spermatozoa. Hum Reprod 1997;12(1):112-118. DOI: 10.1093/ humrep/12.1.112.

10. Jones A, Van Blerkom J, Davis P, et al. Cryopreservation of metaphase Il human oocytes effects mitochondrial membrane potential: implications for developmental competence. Hum Reprod 2004;19(8):1861-1866. DOI: 10.1093/humrep/deh313.

11. Meryman HT. Cryoprotective agents. Cryobiology 1971;8(2):173-183. DOI: 10.1016/0011-2240(71)90024-1. 
12. Nottola SA, Albani E, Coticchio G, et al. Freeze/thaw stress induces organelle remodeling and membrane recycling in cryopreserved human mature oocytes. J Assist Reprod Genet 2016;33(12):1559-1570. DOI: 10.1007/s10815-016-0798-x.

13. Beca JP, Lecaros A, González P, et al. Medical, ethical and legal issues in cryopreservation of human embryos. Rev Med Chil 2014;142(7):903908. DOI: 10.4067/S0034-98872014000700011.

14. Porcu E, Bazzocchi A, Notarangelo L, et al. Human oocyte cryopreservation in infertility and oncology. Curr Opin Endocrinol Diabetes Obes 2008;15(6):529-535. DOI: 10.1097/ MED.0b013e3283199129.

15. Tao T, Zhang W, Del Valle A. Human oocyte cryopreservation. Curr Opin Obstet Gynecol 2009;21(3):247-252. DOI: 10.1097/ GCO.0b013e328329c2d2.
16. Goldman KN, Grifo JA. Elective oocyte cryopreservation for deferred childbearing. Curr Opin Endocrinol Diabetes Obes 2016;23(6):458 464. DOI: 10.1097/MED.0000000000000291.

17. Kushnir VA, Darmon SK, Barad DH, et al. New national outcome data on fresh versus cryopreserved donor oocytes. J Ovarian Res 2018;11(1):2. DOI: 10.1186/s13048-017-0378-4.

18. Mattawanon N, Spencer JB, Schirmer 3rd DA, et al. Fertility preservation options in transgender people: a review. Rev Endocr Metab Disord 2018;19(3):231-242. DOI: 10.1007/s11154-018-9462-3.

19. Lin PY, Huang CC, Chen $\mathrm{HH}$, et al. Failed sperm retrieval from severely oligospermic or non-obstructive azoospermic patients on oocyte retrieval day: emergent oocyte cryopreservation is a feasible strategy. PLoS ONE 2019;14(11):e0224919. DOI: 10.1371/journal.pone. 0224919 . 